\title{
Las connotaciones cognitivas y culturales del bilingüismo
}

\section{The cognitive and cultural connotations of bilingualism}

\author{
Raúl Albino Pesantes Rendón \\ Universidad Internacional del Ecuador, Ecuador
}

Autor para correspondencia: rapesantesre@uide.edu.ec

Fecha de recepción: 20 de julio de 2018 - Fecha de aceptación: 30 de agosto de 2018

Resumen: Este estudio está orientado a analizar la asociación existente entre la lengua, las emociones y la cultura en el proceso de aprendizaje de una nueva lengua y el uso de esta. Para probar la validez de la hipótesis de que los bilingües biculturales sienten y experimentan diferencias en sus respectivas lenguas se realiza un estudio bibliográfico de las diferentes teorías interdisciplinarias de investigadores internacionales. Los resultados apuntan a que las imágenes mentales del bilingüe difieren según la lengua en la que se piensa y que existe una gran variedad de experiencias lingüísticas en las personas bilingües, así como un gran número de variables están presentes en la ejecución de diferentes tareas intelectuales. El objetivo fue definir las características extralingüísticas de los bilingües y los ajustes culturales resultantes de la exposición a dos culturas. Se analizó el lenguaje como una herramienta y se concluye determinando la necesidad de desarrollar y seguir procesos especiales en la educación bilingüe, tomando en cuenta las características sicológicas, culturales y sociales, entre otras, del estudiante bilingüe.

Palabras Claves: bilingües biculturales; emociones; bilingüismo; representaciones mentales

Abstract: This study is aimed to analyze the existing association between language, emotions and culture in the process of learning a new language and the use of it. To test the validity of the hypothesis that bilingual bilinguals feel and experience differences in their respective languages, a bibliographical study of the different interdisciplinary theories of international researchers is carried out. The results suggest that the mental images of the bilingual differ according to the language in which they thought and that there is a great variety of linguistic experiences in the bilingual people as well as a great number of variables are present in the execution of different intellectual tasks. The objective was to define the extra linguistic characteristics of bilinguals and cultural adjustments resulting from exposure to two cultures. Language was analyzed as a tool and concluded by determining the need to develop and follow special processes in bilingual education, considering the psychological, cultural and social characteristics, among others, of the bilingual student.

Key Words: bilingual bicultural; emotions; bilingualism; mental representations 


\section{Introducción}

\section{Bilingüismo}

Hablar es una de las habilidades más útiles que un ser humano adquiere. Esta habilidad se adquiere aparentemente sin esfuerzo, y los niños pequeños a la edad de cinco años ya son capaces de producir frases complejas para expresar sus pensamientos. Como adultos, producimos el lenguaje de forma regular y experimentamos un comportamiento tan automático y no muy exigente (producimos un promedio de unas tres palabras por segundo). A pesar de la facilidad con que producimos el habla, los mecanismos y representaciones involucrados son grandes en número y complejos.

El bilingüismo y multilingüismo, es un hecho importante en la vida cotidiana en el mundo contemporáneo. Para empezar, se habla más de 5.000 idiomas en el mundo en los 194 estados soberanos miembros de la ONU.

Para la comunicación entre los ciudadanos de muchos países del mundo se requiere el bilingüismo y en algunos lugares el multilingüismo. De hecho, David Crystal (1997) estima que dos tercios de los niños del mundo crecen en un ambiente bilingüe.

Considerando sólo el bilingüismo que involucra al inglés, las estadísticas de Crystal, indican que de los aproximadamente 570 millones de personas en todo el mundo que hablan inglés, más del 41 por ciento o 235 millones son bilingües en inglés y algún otro idioma.

Los procesos de globalización en curso sólo pueden aumentar el alcance y el carácter del bi- / multilingüismo, ya que las personas del mundo continúan reconociendo la ventaja de agregar un lenguaje mundial a sus repertorios verbales. Se debe concluir que, lejos de ser excepcional, Muchos investigadores creen, que el bilingüismo / multilingüismo que, por supuesto va de la mano con el multiculturalismo en muchos casos, es actualmente la regla en todo el mundo y lo será cada vez más en el futuro.

Tal vez no es sorprendente que la investigación sobre el bilingüismo ya sea basada en la teoría o la práctica, ha crecido dramáticamente en cantidad, calidad y amplitud en años recientes. Reuniones mundiales han tratado el tema del bilingüismo, estudiando los diferentes aspectos que involucran el hablar otra lengua, presentando los desarrollos en áreas de la investigación teórica y práctica y sus tendencias vitales, que van desde el estudio del cerebro bilingüe, así como el desarrollo cognitivo y cultural del bilingüe. También se ha extendido la investigación al resultado de los estados en la educación bilingüe en diferentes regiones críticas del mundo.

Más de 6000 resúmenes, cada uno de los eruditos contribuyentes desarrollaron investigación en las áreas de su especialización, dados que estos representan campos tan diversos como: Teoría lingüística, neurolingüística, psicolingüística, sociolingüística, discurso y audición, psicología experimental, psicología del desarrollo, Psicología, psicología social, sociología, antropología, educación lingüística, educación de sordos y estudios de área.

La investigación del bilingüismo no es un área de tratamiento del campo, sino un mosaico de enfoques diferentes. 
Estudios del bilingüismo. La investigación está dividida en tres partes, cada una aborda el bilingüismo y sus connotaciones. La Parte II proporciona orientación en el estudio del bilingüismo; Parte III aborda las cuestiones relativas a él individuo bilingüe; La parte IV examina el papel del bilingüismo en la sociedad.

Los fenómenos tratados en este artículo incluyen toda la gama de factores involucrados en el aprendizaje de una nueva lengua, el conocimiento y el uso de dos lenguas su connotación cultural y los cambios en el lenguaje nativo del bilingüe.

Este artículo reúne el trabajo del campo de muchos investigadores y sus aportes bibliográficos compilados en un solo lugar como un aporte para los estudiantes, docentes de inglés y el investigador en bilingüismo, así como aquellos que aplican tal Investigación, espero contribuir con esta investigación bibliográfica a la profundización y ampliación de nuestra comprensión no sólo de las muchas facetas del bilingüismo, sino de los procesos mentales del aprendizaje del estudiante, y la enseñanza aprendizaje de los docentes de la lengua inglesa en general.

\section{Estudio del bilingüismo}

La investigación del bilingüismo es un campo complejo y amplio, que incluye el estudio de la naturaleza del conocimiento del bilingüe individual y el uso de dos (o más) lenguas, así como las consecuencias sociales y culturales más amplias del uso generalizado de más de un idioma en una sociedad determinada. En este capítulo se proporciona una orientación general a este complejo campo. Tomando en consideración "Foundations of Bilingualism", John Edwards (2008), ofrece una visión perspicaz y profunda del campo, examinando una amplia gama de temas que se abordan en este estudio bibliográfico.

Una cuestión importante es preguntarnos ¿quién es un "bilingüe"?, ¿y quién no lo es?, ¿qué relación existe entre el bilingüismo y la inteligencia?, ¿qué influencia tiene el bilingüismo sobre el conocimiento y la cultura? Y una variedad de cuestionamientos a responder con este estudio.

En este estudio se profundizará el bilingüismo del sujeto que puede comunicarse en dos lenguajes, español-inglés o inglés-español. El estudio del bilingüismo colectivo del tipo que se encuentra en la India y en otras sociedades altamente multilingüies plantea su propia gama de cuestiones, incluidas las relativas a la identidad social de los individuos que son miembros de esas sociedades; Edwards también se ocupa de esas cuestiones.

No hay nadie en el mundo que no sabe por lo menos unas pocas palabras en idiomas distintos de la lengua materna, esta competencia, por supuesto, no lo hace bilingüe. Si bien es cierto que un país puede ser bilingüe o multilingüe y, sin embargo, la mayoría de sus ciudadanos pueden tener solamente una sola lengua. Muchos estados de África, por ejemplo, tienen dos lenguas oficiales - usualmente una variedad indígena fuerte y una importante europea para poblaciones altamente heterogéneas y multilingües. Por otro lado, países como Suiza (donde el reconocimiento se concede a cuatro lenguas) o Canadá (oficialmente dos) difícilmente se asemejan a los entornos lingüísticamente ricos y variados de África. Las manifestaciones individuales y sociales del bilingüismo son, por supuesto, variadas. 
Un estudio minucioso del bilingüismo individual implica, por ejemplo, las dimensiones lingüísticas y psicolingüísticas, el nivel social y otras dimensiones de tipo históricas, educativas, políticas, cultural. Etc.

\section{Definición y medición}

Es fácil encontrar definiciones de bilingüismo y estas reflejan respuestas ampliamente divergentes. En 1933, por ejemplo, Bloomfield observó que el bilingüismo era el resultado de la adición de una lengua extranjera perfectamente aprendida a la propia lengua nativa; Sin embargo, prefirió confundir la cuestión admitiendo que la definición de "perfección" era relativa. Posteriormente Weinreich (1953) simplemente definió el bilingüismo como el uso alternativo de dos lenguas; En el mismo año, Haugen sugirió que el bilingüismo comenzó con la capacidad de producir enunciados completos y significativos en el segundo idioma. En general, las definiciones anteriores tienden a restringir el bilingüismo a un dominio igual de dos lenguas, mientras que las posteriores han permitido una variación mucho mayor en la competencia. Pero el argumento de la perfección resulta en la práctica tan insatisfactoria para el propósito de definir el bilingüismo. Se considera, en primer lugar, que hay cuatro habilidades básicas de lenguaje: escuchar, hablar, leer y escribir. Consideremos además las posibles subdivisiones: la habilidad de hablar, por ejemplo, incluye lo que pueden ser niveles bastante divergentes de expresión en vocabulario, gramática y acento. Por lo tanto, hay un número considerable de elementos aquí, todos los cuales figuran en la evaluación del bilingüismo; No se considera que la fuerza en una de estas habilidades signifique fuerza en otra: En general, existen al menos veinte dimensiones de lenguaje que podrían o deberían evaluarse para determinar la proficiencia bilingüe. Se han utilizado muchas pruebas para medir el bilingüismo; Éstas incluyen escalas de calificación y pruebas de fluencia, flexibilidad y dominancia. El primero de ellos puede incluir entrevistas, medidas de uso del idioma y procedimientos de autoevaluación. Podríamos, por ejemplo, pedir a la gente que responda a las instrucciones en dos idiomas, medir sus tiempos de respuesta y, sobre esta base, tratar de determinar la dominancia. O podríamos pedirles a los sujetos que lean en voz alta, o presentar una palabra que coincidan en ambos idiomas (por ejemplo, en inglés y en español) y ver cómo es pronunciado. Podríamos simplemente probar la extensión del vocabulario, o ver cuántos sinónimos para una palabra dada una persona puede llegar a. Sin embargo, aunque los resultados de tales pruebas a menudo están claramente lejos de la perfección.

El segundo idioma puede ser más débil que el primero que, por sí mismo, nunca alcanzará la perfección, ya que todos los factores del lenguaje interactúan fuertemente con las demandas de función y contexto. En algunas circunstancias, el aprendizaje de otra lengua representa una expansión del repertorio lingüístico; En otros, puede conducir a un reemplazo del primero. Los diferentes resultados reflejan diferentes presiones y necesidades sociales.

\section{Adquisición de la competencia bilingüe}

El agregar una segunda lengua no es una tarea fácil, y, sin embargo, la adquisición del segundo idioma se ha dicotomizado como simultánea o sucesiva. La primera describe la exposición a más de una variedad desde el inicio del habla o, al menos, desde una edad muy temprana (algunos profesionales han sugerido la edad tres o cuatro años), mientras que el segundo se refiere a la adición, en una edad posterior. La adquisición simultánea se asocia, por 
ejemplo, cuando un niño habla diferentes idiomas de sus padres. (Incluyen a Jespersen, (1922) ellos sugieren que el desarrollo lingüístico y mental general no se ven afectados negativamente.

La educación de niños bilingües requiere pocos riesgos. Además, cuando se han observado consecuencias negativas, éstas se deben casi siempre a factores sociales, personales, culturales u otros y no al propio proceso de bilingüismo. De hecho, la mayoría de los observadores apuntan a las ventajas de una competencia bilingüe adquirida tempranamente; Esta tiende a reflejar, sobre todo, la relativa facilidad del aprendizaje temprano y los niveles más altos de fluencia, vocabulario, etc.

Hay algunas controversias en cuanto si el bilingüismo comienza desde el nacimiento, o partir de la edad de tres años. Pero la infancia temprana es generalmente mejor que cualquier cosa más adelante (particularmente, quizás, para la habilidad nativa de la pronunciación). A veces se argumenta que el cerebro joven es más "plástico" y "flexible".

Los estudiantes de más edad tienen una experiencia cognitiva que carecen los niños pequeños y, siempre que la motivación sea suficiente, a menudo pueden ser mejores aprendices. Si se pudiera combinar la madurez y la necesidad articulada de los mayores con la impresionabilidad, la imitación, la espontaneidad y la inconsciencia de los más jóvenes, seguramente tendríamos una receta para una adquisición bilingüe rápida y eficiente.

\section{Perspectivas teóricas}

La mayoría de las teorías contemporáneas de la adquisición de un segundo idioma rechazan un enfoque conductista porque ha sido demostrado como inadecuado para comprender el aprendizaje de la lengua materna y una nueva lengua. El aprendizaje ocurre en una serie de etapas no aleatorias, cada una de las cuales se caracteriza por una especie de interlenguaje. Las teorías dentro de la psicología social han prestado especial atención a las características de la motivación, y esto tiene mucho sentido. Si estamos de acuerdo en que el lenguaje es una actividad social, y si aceptamos que casi todo el mundo es cognoscitivamente capaz de aprender, entonces las actitudes que provocan el aprendizaje de una nueva lengua están intrínsecas en el aprendiz. Una distinción hecha por primera vez en los años sesenta indica que la motivación instrumental e integradora para el aprendizaje del segundo idioma es importante. El primero se refiere al deseo de aprender con fines utilitarios, el segundo al aprendizaje de las lenguas como parte de un deseo de conocer más, de interactuar con otras personas, y tal vez en última instancia, sumergirse en otra cultura.

El aprendizaje de una nueva lengua debe incluir al menos alguna motivación integradora. En cualquier caso, un marco bien conocido para el aprendizaje del segundo idioma es el de Gardner (Gardner y Lambert, 1972), que intenta vincular el contexto social, las creencias culturales del aprendiz, capacidades individuales del estudiante e incluir por supuesto los niveles de motivación en el que se va a aprender el idioma.

Una "teoría general" del aprendizaje del segundo idioma ha sido propuesta por Spolsky (1989). Su objetivo es sintetizar esfuerzos tratando de reunir todos los aspectos del aprendizaje de idiomas bajo el mismo techo; Asume que todos los aspectos del aprendizaje son interactivos, todos ellos se interconectan con el tema de la motivación y argumenta que todo aprendizaje de 
idiomas debe ser visto dentro de un entorno social; Sostiene que algunas condiciones para el aprendizaje son "progresivas". Sin embargo, algunos escabullen el mito de que algunas personas, o algunos grupos, no tienen "cabeza" para las lenguas y que la aptitud en la segunda lengua es una mercancía rara que normalmente se ve mejor en los no anglófonos. En su lugar entre lo más importante, se debe tener presente los deseos, necesidades, actitudes, motivaciones de la gente común y los factores sociales que inciden sobre el aprendizaje de idiomas. La simple necesidad es el gran motivador y el gran determinante de hasta qué punto se desarrolla esta competencia.

\section{Bilingüismo e inteligencia}

A menudo se observa que los bilinguies pueden cambiar de un idioma a otro con tanta facilidad y competencia como un conductor de un coche cambia de marcha de un vehículo en condiciones apropiadas. Aunque este comportamiento bilingüe ha sido objeto de investigación lingüística desde hace bastante tiempo, las actitudes generales hacia la mezcla del lenguaje se han basado a menudo en conceptos erróneos fundamentales sobre estos dos aspectos de la competencia lingüística bilingüe. Este capítulo se centra en las cuatro preguntas siguientes sobre el comportamiento verbal bilingüe:

Es una cosa decir que todas las personas normales tienen la capacidad básica de expandir sus repertorios lingüísticos, y que hacerlo no exige ningún precio cognitivo. Pero ¿qué hay de la noción de que el bilingüismo puede aumentar el alcance intelectual? Es una opinión históricamente común que la personalidad crece con lenguas extra, especialmente entre las personas ya bilingües y, más particularmente aún, entre la élite social, para quienes una o dos lenguas adicionales son siempre parte integral de la vida civilizada.

Para los niños que crecen en un ambiente bilingüe y aprenden simultáneamente dos lenguas. ¿Qué supone este esfuerzo para su cerebro? ¿Qué supone esto para la inteligencia? ¿Tal sobrecarga es buena o mala? Pues, en principio, es buena. Es una hazaña formidable Resulta que, al utilizar un idioma, el segundo está también activo, por lo que el cerebro tiene que estar seleccionando entre uno y otro continuamente. Esto produce beneficiosos efectos en la inteligencia, porque refuerza las "funciones ejecutivas", que son la culminación de la inteligencia humana, porque nos permiten dirigir voluntariamente nuestro comportamiento. Activan la memoria de trabajo, fijan la atención, eligen la respuesta, dirigen la acción hacia metas lejanas; y todo esto resulta beneficiado por el bilingüismo, como ha mostrado Albert Costa 2015 y su equipo en la Universidad Pompeu Fabra.

Es curioso ver cómo han cambiado las ideas sobre este asunto. Hasta los años sesenta del siglo pasado, se suponía que los sujetos bilingües presentaban una ejecución inferior en una diversidad de pruebas intelectuales. Poco a poco empezó a imponerse la idea de que no sólo no era un impedimento, sino que se asociaba a puntuaciones más elevadas en tests de inteligencia, y correlaciones positivas entre rendimiento académico y bilingüismo. La capacidad infantil para el aprendizaje lingüístico es pasmosa. Los niños monolingües aprenden con lo que llamamos "principio de exclusividad": cada objeto tiene una palabra. El perro se llama "perro". Pero los niños bilingües desde muy temprano aprenden que tienen, al menos, dos. El perro se llama "perro" y se llama dog. Lo maravilloso es que el niño organiza cada palabra dentro de un idioma, y de acuerdo a la situación utiliza uno u otro sin mezclarlos. 
Hace ya muchos años, Goethe escribió: "Al aprender una lengua extraña, conocemos mejor la nuestra". Es cierto. Usamos con tanta facilidad nuestra lengua materna que no nos damos cuenta de su complejidad, de sus magníficas astucias, de su inaudita eficacia y sutileza. Cuando tenemos que aprender la riqueza de otro idioma, somos conscientes de la belleza del propio. Si bien la diglosia, como bilingüismo colectivo, se considera una condición estable, debe recordarse que incluso la estabilidad es relativa. Un ejemplo es la diglosia franco-inglesa que prevaleció en Inglaterra después de la conquista normanda eventualmente se rompió. Además, la estabilidad de la diglosia puede verse afectada por la presión política y factores nacionalistas y equilibrios entre los grupos étnicos.

\section{El individuo bilingüe}

Características del bilingüe

La mayoría de los investigadores probablemente estarían de acuerdo en que los bilingües, es decir aquellas personas que usan dos (o más) lenguas (o dialectos) en su vida cotidiana, pueden ser caracterizadas por una serie de características generales. En primer lugar, suelen estar influenciados por lo que se ha llamado el principio de complementariedad (Grosjean, 1997), es decir, que usualmente adquieren y usan sus lenguas para diferentes propósitos, en diferentes ámbitos de la vida, con diferentes personas. En segundo lugar, y como consecuencia directa de esta primera característica, los bilingües raramente son iguales en todas las habilidades lingüísticas en todas sus lenguas. El nivel de fluencia depende en gran parte de la necesidad y el uso de un idioma (y de una habilidad particular). Tercero, algunos bilingües todavía pueden estar en el proceso de adquirir un idioma (o habilidad de lenguaje) mientras que otros han alcanzado cierto nivel de estabilidad. En cuarto lugar, el repertorio lingüístico de los bilingües puede cambiar con el tiempo: a medida que el entorno cambia y las necesidades de conocimientos lingüísticos específicos también cambian, también lo será su competencia en estas habilidades. Por último, los bilingües interactúan tanto con monolingües como con otros bilingües y tienen que adaptar su comportamiento lingüístico en consecuencia (ver sección sobre el modo de lenguaje).

\section{Problemas de evaluación bilingüe}

Dos problemas principales se relacionan con la cuestión de la evaluación. En cuanto al primer problema, algunas investigadores todavía sienten que los bilingües tienen o deben tener la misma y perfecta fluidez en cada uno de sus idiomas (lo que se ha llamado los dos monolingües en el punto de vista de una persona, Grosjean, (1997); Otros consideran que el lenguaje se mezcla como una anomalía, ya sea en los niños que adquieren sus idiomas simultáneamente o sucesivamente, o en adultos bilingües; Y otros todavía no recuerdan que muchos bilingües son también biculturales y que sus idiomas reflejarán esta dimensión. Las consecuencias son que pueden hacerse afirmaciones erróneas sobre un comportamiento bilingüe particular, pueden hacerse comparaciones inapropiadas con monolingües y casos excepcionales pueden aplicarse a los bilingües en general.

\section{Soluciones para la evaluación}

Con respecto al primer problema, la falta de comprensión de quiénes son realmente los bilingües, todo lo que se puede decir es que hay un número suficiente de introducciones generales en el campo para ayudar a los investigadores a no caer en esta trampa (véase, por 
ejemplo, Appel and Muysken, (1987). En cuanto al segundo problema, los factores que deben tenerse en cuenta al evaluar, es determinar el campo a evaluar que incluya los tipos de bilingües (bilingües adultos, estudiantes de segunda lengua, niños bilingües, poliglota, etc.) La estabilidad lingüística, la Proficiencia (calificaciones de proficiencia en las cuatro habilidades en los idiomas de los participantes); Gran parte de la información puede ser recolectada a través de cuestionarios por medio de escalas y puede ser reportada numéricamente (tendencias centrales y dispersiones). Hay que considerar las diferencias en la forma en que se evalúan las personas. Por ejemplo, se podría utilizar como un criterio hablantes nativos de un idioma. En segundo lugar, parece crucial distinguir entre los estudiantes de idiomas en un entorno académico que no suelen interactuar socialmente con sus dos idiomas y que por lo tanto no son realmente bilingües (al menos todavía), y las personas que están adquiriendo un lenguaje en un entorno natural y que están usando ambos idiomas en una base regular. Los primeros deben ser caracterizados como "aprendices de idiomas", y tal vez no como bilingües "novatos" o "no fluidos", al menos hasta que empiecen a usar ambos idiomas de manera regular.

\section{Modo activo o pasivo del idioma}

En su vida cotidiana, los bilingües se encuentran en varios modos de lenguaje que corresponden a un continuo monolingüe y bilingüe (Grosjean, 1985a, 1994, 1997a). El modo de lenguaje es una variable que se debe estudiar independientemente (uno deberá investigar las formas de determinar el grado del lenguaje del bilingüe).

Este modo está controlado por variables tales como quién habla, la situación, el tema, el propósito de la interacción, etc. En un extremo del continuo, los bilingües están en un modo de lenguaje totalmente monolingüe en el sentido de que están interactuando sólo con (o escuchando sólo) monolingües de uno -o de otro- de las lenguas que conocen. Un idioma está activo y el otro está desactivado. En el otro extremo del continuo, los bilingües se encuentran en un modo de lenguaje bilingüe, ya que se comunican con (o escuchan) bilingües que comparten sus dos (o más) lenguas y donde puede haber mezclado el idioma (es decir, cambio de código y préstamo). En este caso, ambos idiomas están activos, pero el que se utiliza como el principal idioma de procesamiento (el lenguaje base o matriz) es más activo que el otro.

\section{Diferencias en la interpretación bilingüe}

Las diferencias utilizadas en estudios bilingües, como sílabas, palabras, frases y oraciones, difieren de varias maneras dentro y entre lenguas. Por ejemplo, las palabras pueden diferir en forma gráfica, frecuencia de la forma gráfica, frecuencia y densidad de los pares en la forma gráfica, forma fonética, frecuencia de la forma fonética, frecuencia y densidad de los pares de la forma fonética, categorías sintácticas y frecuencia de estas categorías. Por Así, como puede verse en este caso aparentemente sencillo, las diferencias difieren considerablemente entre lenguas.

\section{Aspectos neurológicos y sicológicos del bilingüismo}

El modelo de memoria declarativa / procedimental

El aprendizaje del lenguaje parece estar mediado por dos mecanismos de memorias diferentes al interactuar con la edad de la adquisición (Paradis, (1994). 
El sistema de memoria procesal es responsable de las funciones motrices y cognitivas adquiridas temprano en la vida. Este sistema está mediado por estructuras de ganglios frontales y basales con contribuciones de regiones parietales inferiores en el hemisferio izquierdo. El aprendizaje de una primera lengua o incluso de una segunda lengua que se aprende temprano en la vida, se basa principalmente en este sistema de cálculo gramatical y procesamiento léxico. El sistema de memoria declarativa media la memoria semántica y episódica y es apoyado por estructuras neurales mediales y temporo-parietales tanto en el hemisferio derecho como en el izquierdo.

El aprendizaje de un segundo idioma más tarde en la vida implica una mayor dependencia de este sistema para el aprendizaje de la gramática, si no el léxico. Por lo tanto, la exposición posterior a un segundo idioma implica un cambio de los sistemas de memoria procedimental a declarativa y una mayor representación del hemisferio derecho. Según los exponentes de este modelo (Fabbro, 2001, Paradis, 1994; Ullman, 2001), Un primer lenguaje tiende a ser aprendido informalmente y en entornos naturales, basándose más en mecanismos de memoria implícita / procedimental y procesos cognitivos inconscientes arraigados en estructuras subcorticales. Los idiomas que se adquieren más tarde en la vida tienden a depender más de la memoria declarativa, a menudo se aprenden en entornos más estructurados y menos naturales, como la escuela, y están representados más exclusivamente en la corteza cerebral que en el primer idioma.

\section{El control inhibitorio entre idiomas}

Los bilingües son capaces de controlar y determinar cuándo usar cada idioma, o cuando un idioma debe ser activado y el otro suprimido o inhibido. Las reglas que determinan la elección de la lengua y los comportamientos de cambio de lenguaje en el cerebro permiten una gran flexibilidad, Sin embargo, una regla general que siempre es observada por los hablantes bilingües y poliglotas es que el cambio de lenguaje tiene lugar sólo cuando el oyente comparte los códigos de idioma que se utilizan. Esta habilidad para cambiar los códigos de lenguaje sólo cuando es contextualmente apropiada es una habilidad que se ha reportado en hablantes bilingües de tan sólo dos años (Leopold, 1949). Se considera hay tres componentes principales en los sistemas lingüísticos de los hablantes bilingües y poliglotas que deben ser explicados para explicar la regulación en el uso de dos o más idiomas.

Cualquier sistema de lenguaje debe abordar primero la cuestión del control. ¿Cómo pueden los hablantes bilingües normales tener acceso a ambos sistemas de lenguaje capaces de controlar qué idioma producen? Si un hablante bilingüe no pudiera separar eficazmente los sistemas lingüísticos, el cambio de código (uso alternativo entre un idioma y otro) violaría muchas de las reglas observadas por los bilingües normales al alternar entre lenguas. El control exitoso de los sistemas de lenguaje implica principalmente evitar errores, como mezclar dos palabras en un idioma o entre lenguas. En los hablantes normales, no ocurre el fracaso de ejercer el control total sobre un sistema de lenguaje intacto, y puede deberse a una variedad de razones, tales como distracción temporal, estrés, fatiga o la influencia de sustancias tóxicas.

El segundo componente en el sistema lingüístico de hablantes bilingües y poliglotas implica la cuestión de la activación determinada por la representación interna de las palabras de las lenguas conocidas. La frecuencia de palabras puede ser influyente en la determinación de la activación o inhibición en los niveles semántico y fonológico de la producción de palabras. Las 
palabras más frecuentes generarán mayores patrones de activación en el acceso léxico que las palabras que son menos frecuentes o familiares. Finalmente, el tercer componente involucra la cuestión de recursos vinculados a procesos de control y regulación, él explica que hay múltiples niveles de control en el procesamiento del lenguaje de los hablantes bilingües.

\section{Adquisición de un lenguaje bilingüismo temprano}

Este enfoca los aspectos del desarrollo del bilingüismo temprano y constituye, en más de una forma, un tema de investigación bastante especial.

La información más importante obtenida de los estudios sobre el bilingüismo infantil en los últimos 25 años es tal vez que la adquisición simultánea de dos o más lenguas puede de hecho ser calificada como una instancia de adquisición múltiple del primer idioma, se afirma que el desarrollo de cada una de las lenguas bilingües procede de la misma manera y conduce al mismo tipo de competencia gramatical que en los respectivos niños monolingües. El desarrollo bilingüe no es cualitativamente diferente de lo que se conoce sobre la adquisición monolingüe, las similitudes y diferencias "cualitativas" se refieren a los aspectos invariantes del desarrollo gramatical. En consecuencia, las preocupaciones principales que emanan de la literatura sobre niños bilingües, así como de las preocupaciones articuladas por padres y educadores, se relacionan con la capacidad de los niños para diferenciar los sistemas lingüísticos, la posibilidad de demoras significativas en la tasa de adquisición del nuevo lenguaje. Sin dejar de considerarse que si el desarrollo lingüístico se demorase más allá de lo que puede considerarse normal investigar si existen afectaciones patológicas. Aunque algunos investigadores informan que los bilingües tienden a comenzar a hablar tarde, es decir, después de los 2 años, 0 (= dos años, cero meses), los retrasos observados están muy dentro del rango de lo que cuenta como una tasa normal de desarrollo lingüístico para niños monolingües. Esto ha llevado a numerosos autores a especular que los bilingües pueden encontrar dificultades, al menos al principio, en separar los léxicos y los sistemas gramaticales de las lenguas que están aprendiendo es que su uso de la lengua normalmente exhibe cierta mezcla.

\section{Adquisición sucesiva del bilingüismo: maduración y edad}

Uno de los principales hallazgos de la investigación sobre el bilingüismo infantil es que la adquisición simultánea de dos o más lenguas puede caracterizarse como una instancia del desarrollo del primer lenguaje en cada uno de los idiomas del niño. Esta declaración, se basa principalmente en el estudio del desarrollo bilingüe en niños que están expuestos a ambos idiomas desde el nacimiento. Sin embargo, es también cierto para los niños que adquieren sus dos o más lenguas sucesivamente es más controvertida. El problema abordado aquí se relaciona con el papel de la edad y maduración en el desarrollo del lenguaje. El tema crucial en el que se basa esta controversia es si la facultad del lenguaje humano o, más precisamente, la "capacidad linguiística" está disponible indefinidamente o si se hace accesible como resultado de la maduración neuronal y permanece accesible sólo durante un período de edad limitado. Si esta última opinión es correcta, se deduce que, si el inicio de la adquisición de otra lengua se produce después de un período crítico.

La predicción es que habrá diferencias cualitativas en el curso de la adquisición, así como en el conocimiento gramatical alcanzado en última instancia, en comparación con las lenguas adquiridas simultáneamente o la primera adquisición lingüística monolingüe. Es importante 
destacar que la existencia de un período crítico para el desarrollo del lenguaje tiene implicaciones significativas no sólo para la adquisición del bilingüismo, sino también para situaciones en las que los niños no tienen pleno acceso al entorno linguí́stico apropiado desde el nacimiento. Los casos más dramáticos son indudablemente aquellos en los que el desarrollo monolingüe se retrasa o interrumpe significativamente cuando los niños crecen aislados. Aunque las preguntas importantes siguen sin respuesta, la evidencia recogida hasta ahora sugiere fuertemente que la capacidad del lenguaje humano está, de hecho, sujeta a cambios maturacionales que resultan en un período crítico para el desarrollo del lenguaje. Por lo tanto, es necesario explorar las consecuencias de la hipótesis del período crítico para la adquisición del bilingüismo.

\section{El constructo de la proficiencia lingüística}

Hasta principios de los años sesenta, los estudios psicométricos tendían a mostrar asociaciones negativas entre el bilingüismo y la inteligencia (Hakuta, 1986). Un estudio de referencia, Peal y Lambert (1962), señaló una serie de problemas metodológicos. Después de hacer los ajustes para estos problemas metodológicos, encontraron una relación positiva entre la inteligencia y el bilingüismo. Desde entonces, varios estudios han reportado los efectos positivos del bilingüismo en la cognición y la metacognición de uno en ambos dominios verbales y no verbales.

Determinar la proficiencia de una persona en dos idiomas es mucho más complicado de lo que parece a primera vista. ¿Cómo podemos medir la proficiencia de alguien en dos idiomas? ¿Cuál es la proficiencia de uno en un lenguaje dado desde el principio? La proficiencia puede ser conceptualizada y medida de varias maneras, reflejando las diferentes visiones que existen hacia lo que el término "lenguaje" designa a sí mismo. Se pueden resumir las principales visiones del lenguaje en tres grupos: (1) la visión lingüística formal; (2) la visión cognitiva y funcionalista; Y (3) la visión sociocultural (Hakuta y McLaughlin, 1996). La visión lingüística se centra en la sintaxis de las lenguas. La visión lingüística formal está fuertemente influenciada por el marco teórico originalmente desarrollado por Chomsky (1965). Al responder al "problema lógico" de por qué los niños pueden adquirir (no simplemente imitar) las lenguas a pesar de su insuficiente aportación, Chomsky propuso que los seres humanos están equipados con una capacidad innata para acceder a un conocimiento inconsciente de gramática. Dependiendo de la exposición del niño a un entorno lingüístico particular, se establecen valores diferentes para un parámetro dado. Se considera que este sistema de principios y parámetros es la base para la adquisición del lenguaje. Es decir, desde este punto de vista, la adquisición del lenguaje se considera un proceso de desarrollo de la gramática de un lenguaje particular mediante la exposición a un entorno inmediato que consiste en el habla en el lenguaje que se adquiere. La distinción de Chomsky entre competencia y rendimiento. La competencia se definió originalmente como "el conocimiento del hablante-oyente sobre el lenguaje", y el desempeño como "el uso real del lenguaje en situaciones concretas" (Chomsky, 1965, p.4). Es importante destacar que lo que él se centró en la investigación fue el conocimiento de los hablantes de las reglas abstractas de la gramática (es decir, la competencia gramatical). En segundo lugar, lo que Chomsky postulaba era la "intuición" del hablante "ideal" de la sintaxis de su primer idioma "de manera completamente homogénea.

\section{El factor edad en la adquisición de un segundo lenguaje (L2)}


A menudo se cree que para adquirir alta proficiencia para ser bilingües equilibrados, uno tiene que empezar a estar expuesto a L2 a una edad temprana. El factor edad es una de las variables más discutidas para explicar las diferencias individuales en la adquisición de L2. Mientras que la adquisición de L1 por lo general comienza al mismo tiempo para todos los estudiantes de L1 (es decir, desde el nacimiento), existe una tremenda variación en cuanto a cuándo los individuos comienzan a aprender un segundo idioma. ¿Apoya la investigación el supuesto de que los niños son más eficientes que los adultos? ¿Es necesario comenzar a aprender la L2 antes de alcanzar cierta edad para lograr una proficiencia similar a la nativa en L2? El factor edad no parece tener mucho efecto. Bailey, Madden y Krashen (1974), utilizando la Medida de Sintaxis Bilingüe, demostraron que sus estudiantes L2 adultos (independientemente de sus antecedentes L1) adquirieron morfemas gramaticales en un orden similar a los niños aprendiendo L2. Sin embargo, estos estudios no están exentos de problemas con su metodología y mediciones, y sus resultados deben interpretarse con cautela. Debido a la falta de estudios longitudinales cuidadosamente diseñados, el efecto de la edad en la ruta de adquisición de L2 no es concluyente en este punto. En cuanto a la tasa de adquisición, hay alguna evidencia de que los adultos superan a los niños cuando la cantidad de su exposición a L2 está controlada [20] (Lowenthal y Bull, 1984; Olson y Samuels, 1973). Además, la ventaja de los estudiantes mayores parecía ser sólo en el corto plazo. Después de una exposición de 10 meses a L2, los niños alcanzaron al grupo de adultos en la mayoría de las medidas. En algunos ámbitos específicos, las ventajas para los niños que aprenden fueron reportadas (por ejemplo, Cochrane, 1980, Tahta, Wood y Lowenthal, 1981), mientras que otras no encontraron diferencias en la tasa de adquisición entre niños y adultos L2 (por ejemplo, Slavoff y Johnson, 1995). Los investigadores se han centrado en el efecto de la edad en la adquisición del segundo idioma, y la cuestión de si hay o no un período crítico para la adquisición del lenguaje y cómo tal período podría afectar ha sido un asunto de debate acalorado. La noción de un período crítico para la adquisición del lenguaje fue propuesta por primera vez por Penfield y Roberts (1959). En otras palabras, la edad de la primera exposición parecía limitar el dominio de ciertos dominios, pero no otros, y los efectos de la edad parecen comenzar mucho antes que la pubertad.

\section{La interacción entre L1 (lenguaje materno) y L2 (segundo lenguaje)}

En la década de 1970, los investigadores adoptaron la visión del papel de L1: el papel de L1 en la adquisición de L2 se consideró mínimo. El análisis de errores reveló que, de hecho, muchos de los errores cometidos por los aprendices no podían ser explicados por la transferencia de L1. Los estudios sobre el orden de adquisición de los morfemas gramaticales, como Dulay y Burt (1974), también indicaron que la orden de adquisición entre L1 y L2 era sorprendentemente similar, independientemente de los antecedentes L1 de los estudiantes.

Recientemente, se ha sugerido que las primeras lenguas de los aprendices no sólo influyen en la adquisición de lenguas secundarias per se, sino que también afectan los procedimientos cognitivos empleados en el procesamiento de las segundas lenguas (Koda, 1997).

\section{Nuevas metodologías de investigación}

En este punto del desarrollo de la investigación del bilingüismo, la metodología de la investigación se ha convertido en una preocupación cada vez más importante, ya es hora de innovaciones en esta área. La razón de esto es que empezamos a tener una noción mucho más clara del papel del procesamiento del lenguaje en la mezcla de códigos y otros procesos de 
contacto y, por tanto, de la relación entre la competencia y el desempeño. Se debe ahondar en las metodologías de investigación, de la lingüística comparada, la lingüística histórica, la lingüística de campo, la sociolingüística y la psicolingüística.

Los psicolingüistas han tomado recientemente en serio la idea de que los bilingües son usuarios de lenguaje más representativos que sus homólogos monolingües. Las investigaciones recientes se centran en cómo los individuos que entienden y hablan más que un solo idioma procesan los límites de dos sistemas de lenguaje que pueden o no compartir características comunes. Sin embargo, otro enfoque igualmente importante es utilizar el bilingüismo como una herramienta para abordar cuestiones sobre la naturaleza de las representaciones mentales y los procesos y los mecanismos de procesamiento del lenguaje en bilingües y estudiantes aprendizaje que los apoyan, la percepción y compresión de palabras y frases la percepción y comprensión cuando los bilingües estén leyendo o escuchando en un solo idioma y también cuando se encuentren en un contexto de lenguaje mixto en el que los dos idiomas puedan ser utilizados indistintamente. Para el propósito de evaluar Tomamos algún nivel de proficiencia para ser bilingüe. Debido a que pocos bilingües tienen un equilibrio genuino en el uso de dos idiomas, suponemos que para la mayoría de los bilingües habrá un idioma dominante, aunque no necesariamente sea el idioma nativo. La mayor parte de la investigación examina el procesamiento del lenguaje en los bilingües que adquirieron su segunda lengua (L2) en algún momento después de la primera lengua (L1) El campo de investigación sobre la adquisición del lenguaje está muy vivo. Aunque se han logrado resultados parciales en ámbitos específicos, queda mucho por hacer tanto conceptualmente como en ámbitos empíricos específicos, la combinación de diferentes.

\section{El papel del bilingüismo en la sociedad}

Esta investigación desvela la gran importancia que tiene el estudio de lenguas dentro del desarrollo de la competencia personal y cultural del individuo. La enseñanza de lenguas extranjeras promueve un acercamiento a una nueva cultura, abre nuevos horizontes y rompe estereotipos. Este hecho permite al individuo ser más tolerante, percibir la realidad de manera más crítica y comprender los fenómenos desde una óptica pluralista e incorporarse a un mundo con perspectiva de globalización.

\section{Bilingüismo individual y colectivo}

La competencia lingüística ampliada suele estar impulsada por la necesidad, pero también se ha reflejado históricamente en diferentes épocas, no haber sabido latín o griego o francés, además de la lengua materna habría sido simplemente impensable para las personas educadas. En otros niveles y por otras razones los ciudadanos más humildes también han sido bilingües desde tiempos remotos por necesidad de comunicarse con quienes les conquistaban o los sometían a trabajos. Existen diferencias importantes entre el bilingüismo individual y el bilingüismo colectivo o social, independientemente de que este último sea o no oficialmente respaldado. El bilingüismo colectivo en muchos contextos, antiguos y modernos es duradero, a diferencia de la variedad transitoria e impermanente muy común en muchos contextos inmigrantes en la generacional en el camino entre dos unilingüismos.

El patrón clásico para los recién llegados a los Estados Unidos, por ejemplo, era el bilingüismo (lengua materna e inglés) para la segunda generación y el monolingüismo inglés 
para la tercera. El bilingüismo colectivo es más permanente y permanece, por supuesto, en gran parte debido a una necesidad continua que no existe entre la mayoría de las poblaciones inmigrantes, y esta necesidad por lo general descansa en diferentes funciones sociales y diferentes dominios de uso para cada idioma. Esta situación se conoce comúnmente como “diglosia". Esta palabra es simplemente la versión griega del bilingüismo (Mackey, 1989), comportamiento verbal real bilingüe implica no sólo nuestro conocimiento de las lenguas en cuestión, sino también una serie de consideraciones de procesamiento. Por lo tanto, tiene sentido, en teoría, separar la competencia de la actuación.

\section{Factores sociopsicológicos: identidad, actitud y cultura}

El contacto de dos o más lenguas dentro de una comunidad de hablantes es una de las múltiples y enriquecedoras consecuencias de un mundo que cada día está más globalizado e interconectado. Desde el punto de vista lingüístico, psicológico y social se han propuesto diferentes explicaciones de lo que es un bilingüe. La definición tradicional (Hammers \& Blanc, 1989) señala que el bilingüe tiene o usa dos lenguas, especialmente habladas con la fluidez característica de un hablante nativo; además, las usa habitualmente y con el control de un nativo. Dominar una lengua no solo consiste en el conocimiento del código lingüístico sino en la capacidad de saber qué decir, cuándo, cómo y en qué contexto decirlo. Lengua y cultura están interrelacionadas y cada día es más importante desarrollar en nuestros estudiantes una competencia intercultural que les permita entrar en contacto con una nueva cultura evitando o, por lo menos amortiguando, las confusiones y malentendidos que pueden darse en la comunicación.

Otro conjunto de factores importantes que influyen en los resultados en la adquisición de L2 es el conjunto de factores sociopsicológicos como la identidad y la motivación que se desarrollan en un determinado contexto de aprendizaje sociocultural. La adquisición del lenguaje está inmersa en sociedades y culturas, y el desarrollo del lenguaje puede considerarse como un proceso de aculturación. Al encontrarse con múltiples grupos y valores culturales y étnicos, los individuos bilingües pueden desarrollar identidades culturales y etnolingüísticas únicas, separadas de las monolingües (Hamers y Blanc, 2000).

En uno de los primeros intentos en esta área, Lambert (1974) conceptualizó las variables socio psicológicas que subyacen al mecanismo para producir las diversas consecuencias del bilingüismo. En su modelo, las actitudes y motivaciones de los estudiantes (junto con la aptitud) influyen en el grado de logro lingüístico. Uno de los logros lingüísticos influye a su vez en la identidad.

Giles y Byrne, 1982). Se han hecho muchos esfuerzos para descubrir los mecanismos sociopsicológicos que subyacen a la adquisición del lenguaje y para identificar las variables influyentes para la adquisición exitosa de L2. Se han propuesto varios modelos, entre ellos el "Modelo Socio educacional" (Gardner, 1979, 1983, 1985), el "Modelo Contextual Social" (Clément y Kruidenier, 1985), el "Modelo de autodeterminación" [21] (Noels, Clément y Pelletier, 1999), y varios estudios empíricos han sido reportados a este respecto. Las actitudes positivas generalmente se encuentran relacionadas con los grados más altos de rendimiento de L2. 
La construcción de la "actitud" en sí varía de modelo a modelo, e incluso dentro del mismo modelo en algunos casos. Por ejemplo, Gardner (1985) distingue la motivación de la actitud donde la motivación se compone de motivación instrumental e integradora. La motivación instrumental se basa en metas funcionales, como conseguir un buen trabajo aprendiendo L2, mientras que la motivación integrativa se basa en un deseo de integrarse en el grupo de lengua y cultura objetivo. Sin embargo, hay un debate estridente sobre qué tipos de motivación están fuertemente relacionados con el rendimiento de L2. Mientras que algunos investigadores afirman que la motivación integrativa está más positivamente correlacionada con el desempeño de L2 (Gardner y Lambert, 1997), otros argumentan un efecto más grande para la motivación instrumental (por ejemplo, Hinkel, 1996, Lukmani, 1972, LoCastro, 2001). Tal vez, como sugiere McLaughlin (1987), la relación entre la actitud y el desempeño de L2 es bidireccional: aquellos que tienen actitudes positivas pueden alcanzar logros más altos, y el logro más alto también contribuye a una actitud más positiva. Además, una perspectiva de desarrollo debe incorporarse más plenamente a estas teorías. Hasta la fecha se han reportado resultados mixtos sobre la relación entre la edad y la actitud (es decir, si las actitudes están positiva o negativamente correlacionadas con la edad). Sabemos poco sobre el mecanismo de cómo los estudiantes forman actitudes, cómo sus actitudes pueden cambiar con el tiempo y cómo tales actitudes pueden afectar la adquisición de L2 en diversos contextos socioculturales.

\section{Conclusiones}

Estos resultados de investigación bibliográfica tienen implicaciones significativas para obtener una mejor comprensión de los procesos de adquisición del lenguaje en los niños y adultos bilingües. Durante los últimos 25 años, investigaciones relacionadas al bilingüismo, han obtenido resultados importantes afirmando que los niños que adquieren dos o más lenguas desde su nacimiento son capaces de diferenciar los sistemas gramaticales de sus lenguas desde muy temprano y sin esfuerzo aparente. Los bilingües adquieren habilidades para usar cada una de sus lenguas, esto incluye la capacidad de conmutar entre lenguajes de una manera sistemática, limitada por requisitos sociales, así como por restricciones gramaticales.

La adquisición de un segundo lenguaje en niños y adultos difiere en aspectos importantes de aquellos casos en los que el inicio de la adquisición ocurre durante los rangos de edad más tempranos. Es cierto que, sin embargo, se necesita mucha más investigación sobre esta cuestión. En cuanto a la adquisición sucesiva del bilingüismo durante los primeros tres o cuatro años de vida, sabemos aún menos. Parece que el inicio de la adquisición durante la edad óptima es una condición necesaria pero no suficiente para el desarrollo de una competencia L1 nativa. La conclusión preliminar que se ha tratado en esta investigación, indica que, debido a la maduración del cerebro, los cambios significativos de aprendizaje ocurren alrededor de los cinco años.

La evaluación del bilingüe es de naturaleza compleja debido a las variaciones individuales en la adquisición de la proficiencia bilingüe. Hay una serie de dificultades para medir con exactitud las proficiencias de los individuos bilingües en ambos idiomas. Los bilingües individuales pueden clasificarse de manera diferente de acuerdo con diferentes dimensiones, tales como las relaciones relativas entre la proficiencia de L1 y la proficiencia de L2, la edad de exposición a un idioma dado y el estatus de un idioma particular en una sociedad dada. La proficiencia puede ser conceptualizada de manera diferente dependiendo de cómo uno 
se aproxima a "los estándares internacionales". Estas evaluaciones pueden captar sólo un pequeño subconjunto de los muchos aspectos del bilingüismo. Es muy importante entender lo que constituye la proficiencia y cómo las lenguas interactúan entre sí. Las evaluaciones tienen que llevarse a cabo en el contexto real y no solo en el contex to académico esto implica incluir los factores de edad, los factores sociopsicológicos, así como condiciones contextuales socioculturales. Al hacerlo, debemos tener en cuenta que aprender otro idioma no es simplemente agregar más conocimientos y experiencias socioculturales. Cada individuo bilingüe desarrollará un perfil lingüístico, cognitivo y sociocultural único, distinto del de los individuos monolingües. La enseñanza de lenguas extranjeras promueve un acercamiento a una nueva cultura, abre nuevos horizontes y rompe estereotipos. Este hecho permite al individuo ser más tolerante, percibir la realidad de manera más crítica y comprender los fenómenos desde una óptica pluralista e incorporarse a un mundo con perspectiva de globalización.

\section{Bibliografía}

David, C. (1997) "English as Global Language second edition. London: Cambridge.

Edward, J. D (2004) “Foundation of bilingualism”. England: OEC Publisging.

Leonardo, B (1993) “Language and Lingüistics”. Chicago: University of Chicago Press.

Uriel, W. (1953 “Language in Contact” N.Y: Linguistic Circle of New York.

Einar, Haugen (1953) Linguistics and Communications”. Philadelphia: University of Pensylvania Press.

Otto, J. (1922) “Language its Nature Development and Origin” London: Routledge.

Robert, G. and Wallace E. (1989) « Attitude, motivation and différences involved in learning English » Canada : Newbury House Publishers.

Bernard, S. (1989) “Conditions for Second Language Learning” U. K: Oxford University.

Annete, G. (2010) Language and Cognition in Bilinguals and multilinguas. New York: Psychology Press.

Rene, A. and Pieter, M. (1987) "Language Contact and Billingualism” U.K. Prifysgol Bangor University.

Michael, P. (1994) "Neurolinguistics of Billingualism and the teaching of Languages". Canada: Department of linguistics, McGill University and Cognitive Neuroscience Centre.

Franco F. (2001 “The Billingual Brain”. Italy: Scientific Institute

Michael T. U. (2001 "Declarative and Procedural Memory” Georgetown: Georgetown University

Noam, Ch. (1965) “Aspect of the Theory of Syntax” Cambridge: MIT Press.

Wilder, P. (1959) “Critical Periods in Language Adqusition” Canada: Mac Gill University. 